\title{
Responding to the Challenge of Early Intervention for Fetal Alcohol Spectrum Disorders
}

\author{
Heather Carmichael Olson, PhD; Tracy Jirikowic, PhD; \\ Deborab Kartin, PhD; Susan Astley, PhD
}

\begin{abstract}
Prenatal alcohol exposure can lead to significant neurodevelopmental disabilities, now recognized as fetal alcohol spectrum disorders (FASD). This includes both fetal alcohol syndrome, a lifelong birth defect, and a wider range of enduring learning and behavior deficits often called alcoholrelated neurodevelopmental disorder (ARND). Diagnostic classification systems have been developed to identify children with FASD, and early interventionists from multiple disciplines can be central in identification and referral for diagnosis, and in providing the known protective influence of intervention early in life. With the recent federal mandates to better address needs of children born prenatally affected by substances, or those impacted by abuse and/or neglect, by referring them for screening and possible early intervention services, there is heightened need for providers to understand FASD. There is a growing body of research data describing the teratogenic effects of alcohol on central nervous system function and physical development, the diversity of children with prenatal alcohol exposure and their families, and the developmental and behavioral characteristics of this clinical population. This article reviews the latest research evidence, bearing in mind what is important to early intervention. This article also gives practical guidance on FASD prevention, methods for early screening, and referral of young children for diagnosis of FASD (and referral for needed services once diagnosed), and how to provide education, support, advocacy assistance, and anticipatory guidance for families raising children with FASD. Key words: early diagnosis and intervention, fetal alcobol syndrome, alcobol-induced disorders (nervous systems), maternal exposure, teratogen
\end{abstract}

From the Department of Psychiatry and Behavioral Sciences, School of Medicine (Dr Carmichael Olson), the Department of Psychosocial and Community Health, School of Nursing (Dr Jirikowic), the Department of Epidemiology, School of Public Health and Community Medicine (Drs Jirikowic and Astley), and Department of Rehabilitation Medicine, School of Medicine (Dr Kartin), University of Washington, Seattle.

This work was also facilitated by grant P3O HDO2274 from the National Institute of Child Health and Human Development.

The authors gratefully acknowledge the Center on $\mathrm{Hu}$ man Development and Disability, Washington State Division of Alcohol and Substance Abuse, and the Centers for Disease Control and Prevention for support during the preparation of this manuscript.

Corresponding author: Heather Carmichael Olson, PhD, Child Psychiatry Research, 1100 Olive Way, Suite 800, Met Park West Bldg, Children's Hospital Child Psychiatry Research, Seattle, WA 98101 (e-mail: quiddity@ u.washington.edu)
DREnatal alCOHOL EXPOSURE can lead to significant developmental disabilities, now recognized under the umbrella term of "fetal alcohol spectrum disorders" (FASD) (National Organization on Fetal Alcohol Syndrome, 2004). The number of children with the full "fetal alcohol syndrome" (FAS) has been estimated to be 0.5 to 3 per 1000 live births, with higher rates in some communities (Stratton, Howe, \& Battaglia, 1996). But FAS is found in only a fairly small proportion of children affected by prenatal alcohol exposure. Many children have alcoholinduced impairments that can be just as serious, or more so, than those seen in FAS (Mattson, Riley, Gramling, Delis, \& Jones, 1998). The term "alcohol-related neurodevelopmental disorder" (ARND) has been applied to this condition (National Institute on Alcohol Abuse and Alcoholism [NIAAA], 
2000). Prevalence rates of the full range of FASD beyond FAS, including ARND and other alcohol-related birth defects, are believed to occur about 3 times as often as FAS. A rate of 2 to 6 per 1000 (Centers for Disease Control and Prevention [CDC], 2007), and approach the latest estimated prevalence of autism spectrum disorders. New regulations at the federal level, under both the Keeping Children and Families Safe Act of 2003, which amended and reauthorized the Child Abuse Prevention and Treatment Act (CAPTA), and the Individuals with Disabilities Education Improvement Act of 2004, require that children involved in substantiated cases of child abuse and neglect be referred to early intervention systems. IDEA further requires early intervention referral for children whose development is impacted by prenatal substance exposure. These regulations are certain to result in increased numbers of children in early intervention systems who need referral for FASD diagnosis followed by tailored early intervention.

Clearly, FASD is an important problem that every early interventionist will encounternow more than ever-and potentially play a key therapeutic role. To help guide practice in early intervention, this article summarizes current research on FASD and provides practical ideas about prevention, qualifying children for services, supporting families, anticipatory guidance, and a developmental systems model useful in thinking about assessment and intervention for young children with FASD.

\section{ALCOHOL AS A TERATOGEN AND FASD PREVENTION}

As a neurobehavioral teratogen, alcohol interferes with normal fetal growth and development through multiple actions at different sites. Researchers are now studying the biochemical mechanisms underlying alcohol's effects, in hopes that pharmacologic treatments might eventually be used to intervene with (or prevent) alcohol-related fetal injury. Researchers are also looking at neuroimaging and physical measures (such as EEGs) to understand more precisely how alcohol damages the brain (NIAAA, 2000). Most important to early interventionists is the fact that prenatal alcohol exposure can significantly compromise central nervous system (CNS) function, leading to a wide range of developmental, learning, and behavior problems. These problems can affect later life success. Indeed, natural history data so far show that individuals with FASD have a very high likelihood of suffering secondary disabilities of lifestyle and daily function in adolescence and adulthood (Streissguth et al., 2004).

Fetal effects of alcohol exposure differ depending on the amount, timing, and pattern of maternal drinking, so deficits are highly variable from one child to another. In general, the more a pregnant woman drinks, the greater the severity of persistent neurobehavioral deficits. Episodic (or binge) drinking that creates higher maternal peak blood alcohol concentrations is associated with greater fetal damage. On an individual basis, however, any amount of drinking during pregnancy can cause harm, and alcohol use at any time during gestation is associated with a higher risk of CNS dysfunction. Figure 1 provides highlights from the 2005 U.S. Surgeon General's Advisory on Drinking and Pregnancy (2005). Early interventionists can advance the important goal of FASD prevention by providing this information to the many women with whom they work who are thinking about pregnancy or are already pregnant, and to women's partners.

\section{IDENTIFYING THE PROBLEM AND QUALIFYING CHILDREN FOR SERVICES}

Table 1 defines diagnoses on the fetal alcohol spectrum, and Figure 2 shows the "face" of FAS. These diagnoses are medical conditions. Current practice guidelines suggest that diagnosing physicians work with a multidisciplinary team and follow welldefined FASD diagnostic guidelines. National diagnostic guidelines have been published in the United States for FAS (National Center 


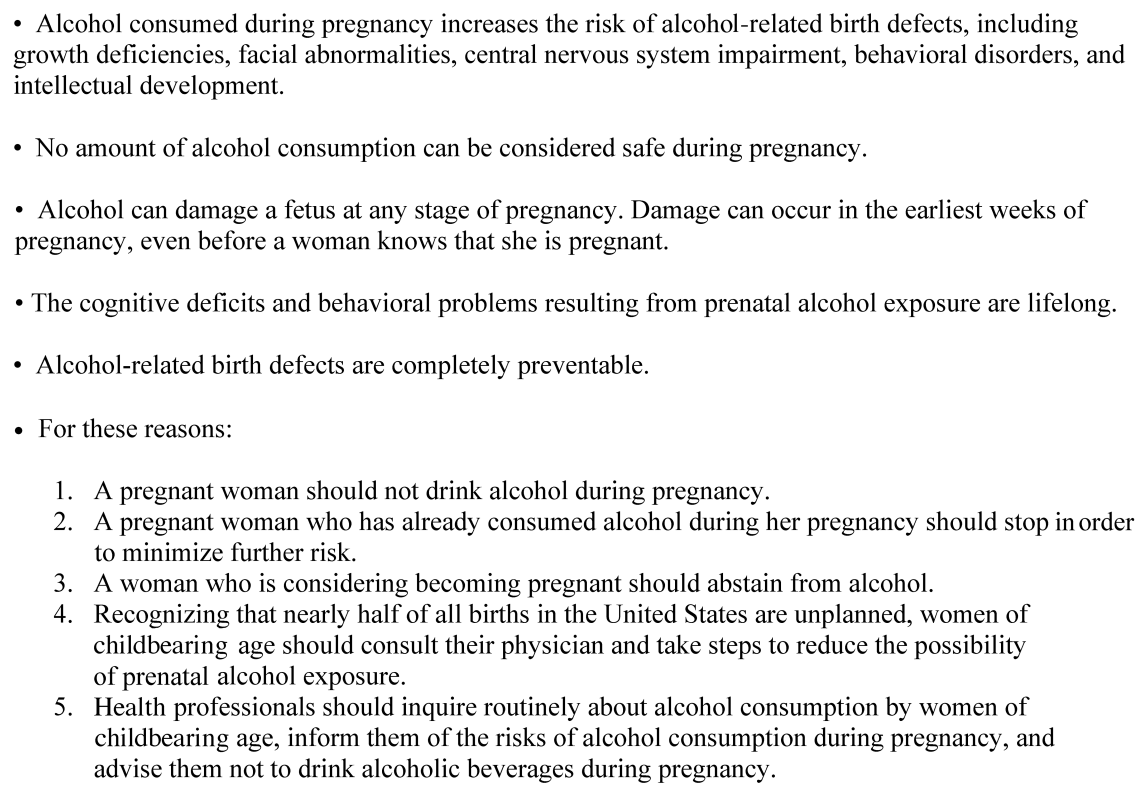

1. A pregnant woman should not drink alcohol during pregnancy.

2. A pregnant woman who has already consumed alcohol during her pregnancy should stop in order to minimize further risk.

3. A woman who is considering becoming pregnant should abstain from alcohol.

4. Recognizing that nearly half of all births in the United States are unplanned, women of childbearing age should consult their physician and take steps to reduce the possibility of prenatal alcohol exposure.

5. Health professionals should inquire routinely about alcohol consumption by women of childbearing age, inform them of the risks of alcohol consumption during pregnancy, and advise them not to drink alcoholic beverages during pregnancy.

Figure 1. Highlights of the 2005 surgeon general's advisory on drinking and pregnancy. From http:// www.surgeongeneral.gov/pressreleases/sg02222005.html.

on Birth Defects and Developmental Disabilities [NCBDD], 2004) and in Canada for the broader spectrum (Chudley et al., 2005). More specific FASD diagnostic systems have been developed for clinical and research use which provide a method for diagnosing the full range of conditions that make up the fetal alcohol spectrum.

FASD diagnoses commonly co-occur with developmental delays or deficits arising from other genetic or medical causes, psychiatric disorders (such as ADHD), and/or academic problems. A full understanding of an individual child's problems requires taking all co-occurring conditions into consideration. Diagnoses on the fetal alcohol spectrum typically add an important dimension to the description of a child's problems as classified by other medical and psychiatric diagnoses generated through the use of diagnostic systems such as $I C D-10$ (World Health Organization, 2005), DC:0-3-R (Zero to Three, 2005), or DSM-IV (American Psychiatric Association, 1994). Knowing that a child has ADHD in the presence of FASD, for example, can mean that commonly used interventions for ADHD will not have expected effects, and that a dif- ferent combination of treatment techniques may be required. Indeed, an understanding that a child has neurological unpairment arising from alcohol exposure can fundamentally change a parent's or provider's perception of the child's behavior.

Current FASD diagnostic guidelines usually require evidence of structural, neurologic, and/or functional damage of the CNS. Evidence of structural/neurologic damage may include such findings as microcephaly (small head size), abnormal neuroimaging, or seizure disorders. These can sometimes be identified early in life. Evidence of CNS dysfunction includes standardized test scores that document significant global delays or, more commonly, variability revealed in significant gaps in skills, atypical patterns of development, or uneven profiles of learning strengths and weaknesses. Unfortunately, it is often difficult to gather clear evidence of CNS dysfunction at a young age. Early developmental deficits of alcohol-exposed children may be subtle and yet important precursors of later problems. Tests used with young children often cannot detect variability in learning profiles or subtle problems, so young children 
Table 1. Criteria for diagnosing fetal alcohol spectrum disorders ${ }^{*, \dagger}$

\section{Diagnosis}

Fetal alcohol syndrome (FAS)

Partial FAS (PFAS)

Alcohol-related neurodevelopmental disorder (ARND)

\section{Diagnostic features}

Growth deficiency: Height or weight less than 10th percentile

Cluster of characteristic minor facial anomalies: Small palpebral fissures (eyeslits), thin upper lip, smooth philtrum (groove above the upper lip)

Central nervous system damage (evidence of structural and/or functional brain impairment)

Reliable evidence of confirmed prenatal alcohol exposure: Not necessary if the cluster of characteristic facial features is fully present

Some of the characteristic minor facial anomalies

Growth deficiency: Height or weight less than 10th percentile

Central nervous system damage (evidence of structural and/or functional brain impairment)

Reliable evidence of confirmed prenatal alcohol exposure

Central nervous system damage (evidence of structural and/or functional brain impairment)

Reliable evidence of confirmed prenatal alcohol exposure

\footnotetext{
*Adapted from the Astley (2004).

${ }^{\dagger}$ The 4-Digit Diagnostic Code (Astley, 2004) is a system used by clinicians and researchers to evaluate the effects of prenatal alcohol exposure. While the 4-Digit Code maps onto these widely known diagnoses, the system actually uses different descriptive terms to more precisely describe bow children manifest PFAS and ARND. There are other diagnostic guidelines that also include a category called "Alcohol-Related Birth Defects" (ARBD). ARBD exist in the presence of confirmed prenatal alcohol exposure, but children who have ARBD do not necessarily show the characteristic facial features. ARBD are defined as follows: "Any of a number of anomalies (such as heart or kidney defects) present at birth that are associated with maternal alcohol consumption during pregnancy” (NIAAA, 2000, p. 286).
}

will less often meet FASD diagnostic criteria. In addition, because alcohol effects may often emerge most clearly as deficits in higher level cognitive functions, children's problems may not even become evident until well past the window for early intervention, around second to fourth grades (Olson, Morse, \& Huffine, 1998b). Yet there is growing knowledge about the plasticity of the CNS, and intriguing findings on the positive developmental effects of enriching the motor and learning environment with alcohol-exposed animals (eg, Klintsova, Goodlet, \& Greenough, 1999; Miura, Whinery, Dominguez, Riley, \& Thomas, 2005). These strongly imply that early identification and intervention for children who are alcohol-exposed may be especially important, because CNS function might potentially be improved.
Some states recognize the full FAS as a diagnosed condition with a high probability of developmental delay, thus deserving early intervention. But there are many additional children impacted by prenatal alcohol exposure, and new diagnostic guidelines recognize conditions across the full fetal alcohol spectrum. Literature review later in this article strongly indicates that children affected by prenatal alcohol, but without the full syndrome, have diagnosable conditions that also carry a high likelihood of developmental delay and later significant problems in adaptive function. Because of this, providing early intervention services for all children diagnosed with FASD should be strongly encouraged. An even broader approach to qualifying developmentally vulnerable young children for early intervention is to classify them "at-risk" 


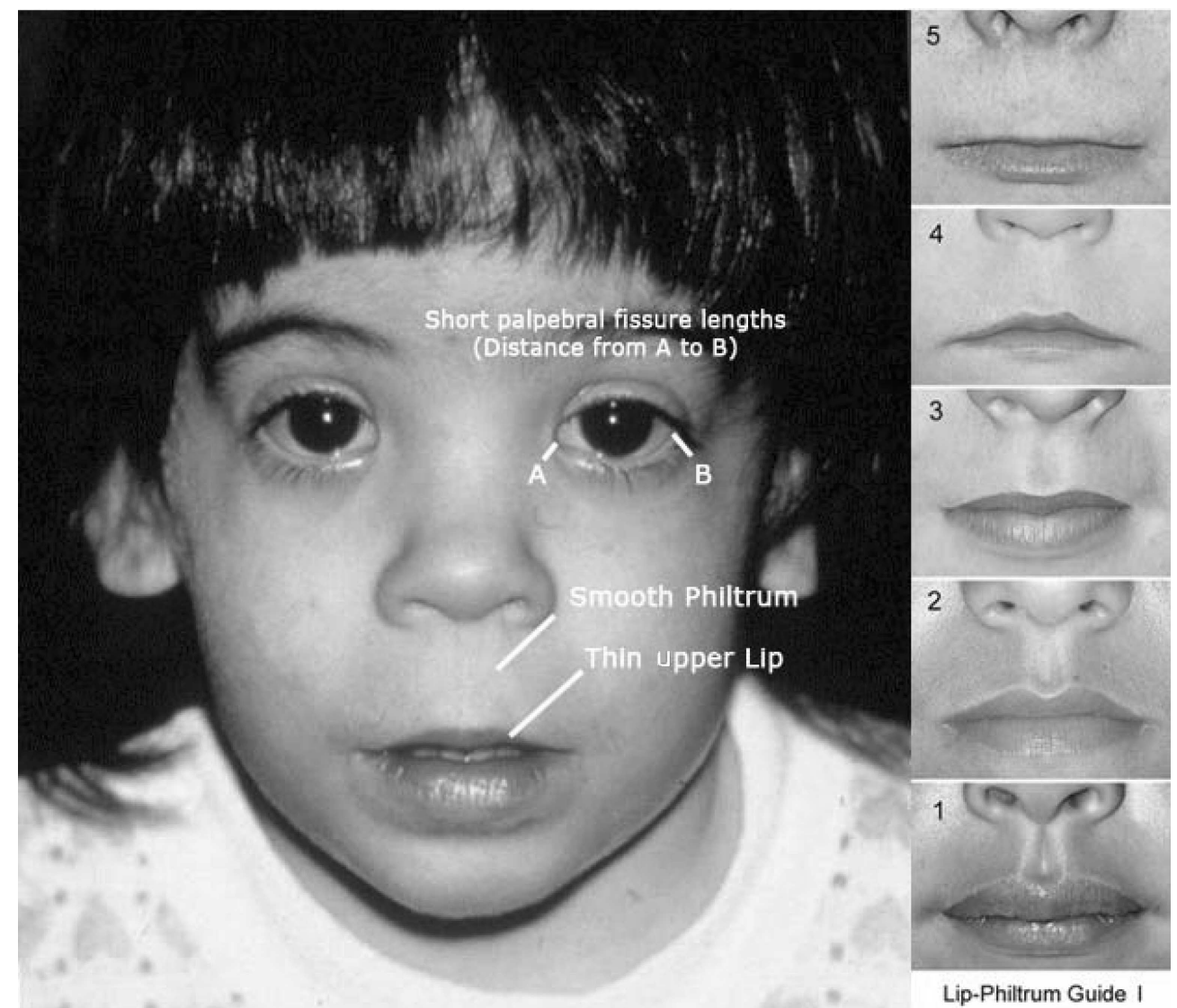

Figure 2. The 3 diagnostic facial features of fetal alcohol syndrome are as follows: (1) palpebral fissure length (eyeslit length) of 2 or more SD below the mean; (2) smooth philtrum (the vertical groove between the nose and the upper lip) (rank 4 or 5 on Lip-Philtrum Guide); (3) thin upper lip (rank 4 or 5 on Lip-Philtrum Guide). Used with permission from Susan Astley.

because of prenatal alcohol exposure coupled with evidence of emerging learning problems and/or environmental risk if this approach fits into IDEA implementation in the state where the child resides. One strategy that does not fit with current evidence is assuming that an alcohol-exposed child will "grow out of" apparently mild early delays, and therefore not providing services or developmental monitoring. Instead, if an exposed child does not qualify for early intervention, or improves after intervention and then no longer qualifies, a better approach lies in careful monitoring and reevaluation at key developmental transitions. This will address the possible emergence of later cognitive, language, and social difficulties.

\section{YOUNG CHILDREN WITH PRENATAL ALCOHOL EXPOSURE AND THEIR FAMILIES}

What does current scientific evidence say about young children with prenatal alcohol exposure, and what are the implications for early intervention? At present, data for young children born prenatally exposed to alcohol come from several well-designed prospective longitudinal studies of the impact of moderate or higher levels of prenatal alcohol exposure conducted in various locations in the United States, Canada, and selected other countries (with varying ethnic compositions and levels of environmental risk). There are also a few small but carefully investigated clinical 
samples of young children with FASD. Just now emerging are data from larger clinical samples composed of patients seen in FASD diagnostic clinics. All these studies are the source of research findings reviewed here, given the focus of this article on the direct impact of prenatal alcohol exposure, a proven neurobehavioral teratogen, on child and family outcome. Also available in the literature are longitudinal studies of young children born polydrug-exposed, including alcohol, who typically have lives characterized by high levels of postnatal environmental risk. Findings from these latter studies are referred to briefly but not reviewed here, because these studies primarily provide insight into the impact of illicit drug exposure coupled with the larger issues of very high risk families and the impact of chemical dependency, rather than the impact of alcohol exposure before birth.

To begin, we briefly summarize new data on a large number of young children, aged birth to 8 , and their families $(N=781)$, from the Washington State FAS Diagnostic and Prevention Network (FAS DPN). The FAS DPN is a statewide clinic network operating since 1993 that uses an interdisciplinary team diagnostic process and a carefully developed diagnostic system called the "4-Digit Diagnostic Code" (Astley, 2004; Astley \& Clarren, 2000). The FAS DPN data discussed here come from the largest clinical database of children born prenatally alcohol-exposed believed to be in existence at this time, and includes a large number of young children. There are no national data on this set of neurodevelopmental disabilities, so the FAS DPN database is an excellent source of information useful to early interventionists even though conclusions are somewhat limited by geographical context. Compared to the population of Washington State, the overall FAS DPN database includes somewhat fewer females and, in terms of ethnicity, slightly underrepresents Caucasians (including those of Hispanic origin) and slightly overrepresents African Americans. Largely because of referral patterns (perhaps because of cultural differences in recognition of the prob- lem and willingness to refer), the FAS DPN database includes somewhat more Native Americans and fewer Asians than in the larger population of Washington State. It should be noted that the FAS DPN database likely underrepresents the subset of children with FASD still living with parents grappling with chemical dependency. Research is needed on the prevalence of FASD in this high-need group.

\section{Age of identification and important demographic characteristics}

FAS DPN data show that the average age of referral for FASD diagnosis overall is $9^{1 / 2}$ years. This means that identification of problems related to alcohol exposure often occurs rather late in children's lives. Younger children, aged birth to 8 , comprise only half of those referred. Of these, the average age of identification is just more than 5 , with only about a third brought in before age 4 . This indicates that two thirds of younger children missed the chance for true early intervention. Improved recognition of FASD by early interventionists could bring more young children in for early diagnosis. Early diagnosis has been identified as a crucial protective factor in this population, because it is associated with more positive outcomes in adolescence and adulthood (Streissguth et al., 2004).

Many children referred for FASD diagnosis are not in the care of their birth parents. In the FAS DPN database, more than $70 \%$ of those referred for FASD diagnosis are in foster/adoptive homes. Interestingly, almost half of young children referred (and those diagnosed with FAS or Partial FAS [PFAS]) are at least third born (or later), which fits with observations that the impact of prenatal alcohol exposure may be greater for later borns. Children referred for FASD diagnosis have various racial and ethnic backgrounds. Half of those referred (and those diagnosed with FAS/PFAS) are considered Caucasian, fewer than expected given Washington State demographics, while the others come from a variety of ethnic backgrounds. 


\section{Need for referral for FASD diagnosis and advocacy by early interventionists}

Surprisingly, in the FAS DPN database, very few families (4.7\%) of young children are referred for FASD diagnosis from school or early intervention settings, but instead come from other referral sources such as medical, psychological, or social service providers. This low referral rate could be raised by increased awareness among early intervention providers of referral signs and the hazards of alcohol exposure, and by recognizing the importance of subtle early deficits when prenatal alcohol exposure is present (which even the children's parents may not fully appreciate).

Young children in this database receive FASD diagnoses across the whole spectrum, at rates consistent with population-based studies. This fits with wide variation in prenatal alcohol exposure seen in this population, ranging from maternal consumption of a single glass of wine once in pregnancy to daily intoxication throughout pregnancy. Of children aged birth to 8 , only $7.4 \%$ receive an FAS or a PFAS diagnosis. It is this surprisingly small group who has the "face" of FAS and who is most likely to be recognized and qualify for early intervention. This is true even though their CNS dysfunction is often no more severe than that of children with ARND. A larger percentage (25.0\%) of young children are diagnosed with the equivalent of "severe" ARND. While these young children are clearly in need of intervention, their families may not be able to access help without strong advocacy from early interventionists. An even larger percentage (43.4\%) are diagnosed with the equivalent of "mild" ARND. These youngsters may very well show significant problems later on. Yet they are far less likely to receive any early intervention unless providers are very well informed, and have the chance to serve these children within an "at-risk" category.

\section{Early indicators suggesting referral for an FASD diagnosis}

FAS DPN data suggest that the simple presence of prenatal alcohol exposure may be the most important reason to refer for diagnosis, with higher levels of exposure especially worrisome. Behavior problems in the presence of prenatal alcohol exposure are also an important referral sign. FAS DPN data also reveal that some indicators commonly used by providers to prompt early identification of problems may not be as useful in signaling the need to refer for FASD diagnosis. For example, the average gestational age for children referred to FAS DPN clinics is very close to full term at 37.3 weeks. As another, very striking example, only just over half of the children show marked developmental delay in the first 3 years of life-even among those who receive an FAS/PFAS diagnosis. Indeed, more than one fourth of referred children have developmental profiles well within normal limits, with an additional third who show only mildly abnormal developmental scores. Some familiar indicators of early risk do characterize this group. Data from the FAS DPN show that most of these young children (84.5\%) are also exposed to cigarettes or illicit drugs, and most (82.6\%) have experienced some degree of postnatal environmental risk.

FAS DPN data reveal that some important early signs for referral for FASD diagnosis that providers could use, with training, are often being missed. It was surprisingly rare for young children to be referred for concerns about facial anomalies or growth. Indeed, diagnostic referral because of FAS-like facial features occurred for only about a tenth of these young children. Yet with careful diagnostic measurement about three quarters of young children referred showed at least 1 or more of the "sentinel" physical features. If early interventionists were more aware of these characteristic features, and/or if facial features were regularly screened as part of an early intervention intake process, diagnostic referral might occur more often. FAS DPN data do suggest that it remains important to pay close attention to even mild impairments of body growth and, especially, to small head size ( $\geq 2$ SD below the mean). Children with microcephaly show more developmental problems than the general population (Dolk, 1991), and over 
two thirds of young children diagnosed with FAS/PFAS had microcephaly.

\section{THE EFFECTS OF PRENATAL ALCOHOL EXPOSURE ON DEVELOPMENT IN YOUNG CHILDREN}

\section{An overview, including problems in sensory processing}

What is known about the effects of prenatal alcohol exposure on development in young children? It is well established that heavy levels of prenatal alcohol exposure lead to neurobehavioral deficits, but the effects of lower levels of alcohol exposure are less clear (NIAAA, 2000). Neurobehavioral deficits arising from alcohol exposure are lifelong, but their impact on adaptive function emerges more clearly over time-and can likely be improved or made worse by postnatal experiences.

A good overview of clinically relevant findings is provided by a recent, careful study using FAS DPN data that compare a clinical sample of twenty-five 5- to 8-year-olds to an age-, gender-, and ethnicity-comparable group of 26 typically developing peers (Jirikowic, 2003). Children with FASD performed significantly more poorly than comparison peers across measures of IQ, adaptive behavior, academic performance, and sensory-motor development. A main contribution of this study was findings of sensory processing problems in the children with FASD, which validated and extended findings of an older nonstandardized questionnaire study (Morse, Miller, \& Cermak, 1995). Using a standardized parent questionnaire coupled with direct testing, Jirikowic (2003) found clinically significant difficulties among children with FASD in tactile, auditory, and visual sensitivity, underresponsiveness to sensory information and sensation-seeking behaviors, and auditory filtering. Although as a group overall motor performance was in the low average range, specific difficulties were seen in sensory-motor tasks involving eye-hand coordination and timed responses, and on tests revealing persistent minor neurologic soft signs. Children with FASD showed generally lowered early academic performance (though still in the average range), with significantly low mathematics skills and early spelling skills. Overall adaptive level was lower than among children with typical development, but quite variable across alcohol-exposed individuals. For children with FASD, relative strengths in adaptive behavior were seen in ratings of motor function, and poorer performance in socialization and ability to follow the rules of daily living. Both teachers and caregivers also endorsed significantly more behavior problems among children with FASD than among typically developing peers. Interestingly, analysis of this carefully constructed and diagnosed sample revealed no differences in performance between girls and boys in the group with FASD. Gender differences have almost never been assessed in other studies.

\section{Problems in early cognitive skills and learning, and sleep}

Although there is a wide variety in the type and extent of deficits, problems in cognition, learning and memory, attention, and academic achievement have consistently been found among preschool (eg, Janzen, Nanson, \& Block, 1995; Jirikowic, 2003) and older children with FASD (eg, Coles et al., 1997; Howell, Lynch, Platzman, Smith, \& Coles, 2006; Mattson \& Riley, 1998). In clinical samples with FASD, there are consistent findings of deficits in attention, wide-ranging difficulties in higher order cognitive processes called "executive functions," visual-spatial processing deficits and other problems in information processing speed and efficiency, and difficulties with mathematical problem-solving and achievement (NIAAA, 2000). Also of special interest are newer findings of alcohol effects on basic conditioned learning and other cognitive functions that reveal an impact on specific brain regions (such as the cerebellum) (eg, Jacobson et al., 2005). An interesting emerging area is potential alcohol effects on sleep, with likely sleep difficulties in respiratory control and/or circadian rhythms related to problems in brain development, and also to physical problems in midface development 
leading to possible breathing obstruction (M. Chen, personal communication, January 3, 2006).

Prenatal alcohol exposure has been associated with cognitive and learning difficulties into adolescence (eg, Goldschmidt, Richardson, Cornelius, \& Day, 2004; Streissguth, Barr, Sampson, \& Bookstein, 1994) and beyond, though not all longitudinal studies find effects (Greene et al., 1991). Prenatal alcohol has been associated with mildly decreased performance on the Bayley Mental Scale in infancy (eg, Jacobson et al., 1993) and with mildly lowered IQ scores in preschoolers (eg, Streissguth, Barr, Sampson, Darby, \& Martin, 1989). Newer longitudinal research with wider ethnic diversity and somewhat more highly exposed samples (using more sensitive measures) has found an association between alcohol exposure and early, specific cognitive and achievement difficulties. For example, infants prenatally exposed to alcohol have shown deficits in information processing and efficiency (Jacobson, Jacobson, \& Sokol, 1994). These persist into the early elementary years, with additional findings of a distinctive prenatal alcohol effect on number processing (Burden, Jacobson, \& Jacobson, 2005).

\section{Deficits in speech, language, and communication}

A variety of speech, language, and communication problems have been described for clinical samples of school-aged children with heavy prenatal alcohol exposure, including delays in speech acquisition, impaired receptive and expressive language, and problems in speech production (Church \& Kaltenbach, 1997). Some school-aged children with FASD show discrepancies between relatively better verbal abilities (eg, vocabulary, basic syntax), and diminished capacity to use these skills effectively in social communication (which is an important foundation for developing social relationships and exchanging information) (Coggins, Friet, \& Morgan, 1998). There has so far been limited clinical or longitudinal study of language and communication in infants and young children with prenatal alcohol exposure, although this is an area of intense research interest. Current clinical thinking is that young children with FASD can often acquire basic linguistic skills in a typical manner, but have difficulty becoming socially competent communicators later on and, as they grow older, show deficits in higher order language skills. These include difficulties in the more complex syntax, metacognitive abilities, and narrative skills that are tied to their cognitive difficulties in executive functions (eg, Coggins, Olswang, Carmichael Olson, \& Timler, 2003).

\section{Problems in motor development and neurologic soft signs}

Alcohol-exposed individuals consistently show impairments in the development of motor control (NIAAA, 2000). In infancy, clinical studies reveal motor delays occurring more often and/or with increased severity in the presence of heavy alcohol exposure (eg, Autti-Ramo \& Granstrom, 1991). Among younger children with FASD, delays in motor skills are generally mild to moderate in extent, and poor movement quality (decreased speed, efficiency, and control; abnormal balance) is often seen (eg, Osborn, Harris, \& Weinberg, 1993). Significant deficits in visualmotor development (but not in motor-free visual perception), and in fine motor coordination, have been described in samples of younger children with FAS in comparison to that of typically developing peers (Adnams et al., 2001; Janzen et al., 1995).

Some longitudinal researchers have found no discernable impact of more moderate levels of social drinking on motor development in infancy (eg, Richardson, Day, \& Goldschmidt, 1995) or early childhood (eg, Chandler, Richardson, Gallagher, \& Day, 1996; Fried \& Watkinson, 1990; Larrouque \& Kaminski, 1998). Other long-term studies have found clinically significant effects, but only among infants at high exposure levels-with qualitative differences in motor behaviors (eg, deficits in imitating movements, standing, and walking) noted in infants 
with lower exposure levels when compared to those with no exposure (Jacobson et al., 1993). In early childhood, longitudinal research has identified increased levels of minor neurologic soft signs among alcohol-exposed preschoolers (Larrouque et al., 1995), and a positive association between greater alcohol exposure and increased deficits in fine motor steadiness and balance (Barr, Streissguth, Darby, \& Sampson, 1990).

\section{Difficulties in adaptive behavior and social-emotional development}

Difficult behaviors and social skill deficits that persist across time are an overarching concern among individuals of all ages with prenatal alcohol exposure in both clinical and longitudinal samples (eg, Olson et al., 1997; Olson, Feldman, Streissguth, Sampson, \& Bookstein, 1998a; Spohr \& Steinhausen, 1996). Mental health problems have been reported for a very large majority of individuals with FASD in natural history research (Streissguth, Barr, Kogan, \& Bookstein, 1996), and elevated rates of psychiatric disorders are a concern (eg, Fryer, McGee, Matt, Riley, \& Mattson, 2005; Murray, Olson, \& Montague, 2005; O'Connor, Kogan, \& Findlay, 2002a). For school-aged children with FASD, studies using questionnaires and child behavior checklists often show clinically elevated attention, social, and sometimes internalizing problems (eg, Mattson \& Riley, 2000; Roebuck \& Riley, 1999), and sometimes also problems with externalizing behavior and aggression (eg, Olson, Brooks, Davis, \& Astley, 2004). Adaptive behavior and social skills among pre-school-aged and young school-aged children with FASD are reported as lower than expected for age and intellectual level (Mattson, Lee, Hayden, \& Riley, 2005; Thomas, Kelly, Mattson, \& Riley, 1998). It appears that children exposed to alcohol show relatively greater deficits in adaptive function than do clinic-referred peers after the age of 8 (Whaley et al., 2001). Clinical studies of children with FASD use terms such as impulsive, distractible, and "always on the go" to describe behavior in the preschool and elementary years (Janzen et al., 1995). In addition, while younger children with FASD have been described as engaging, verbal, apparently alert, and "bright-eyed" (Streissguth, 1997), and appear more functional than they actually are, they seem to lack social boundaries (Olson, 1994). There is wide variability in the level of these deficits, ranging from subtle to severe.

Longitudinal studies focused on infancy report early problems in behavioral regulation associated with prenatal alcohol exposure, including mild to moderate irritability, poor habituation, sleep problems, and feeding difficulties (Eyler \& Behnke, 1999; Streissguth, Barr, Martin, \& Herman, 1980). Among preschoolers, limited longitudinal data suggest that prenatal alcohol exposure has been associated with functional compromise that includes mild to moderate inattention and hyperactivity, and subtle to moderate impulsivity, and behavior problems (eg, Streissguth, Bookstein, Sampson, \& Barr, 1993, 1995).

Developmental systems thinking adds to our understanding of the direct "main" effects of alcohol on child outcome, to better explain the reverberating impact of prenatal alcohol exposure and maternal drinking on socialemotional development across infancy and early childhood. O'Connor and her colleagues (eg, O'Connor et al., 2002b) described a transactional process in which alcohol-exposed infants show increased negative affect, their mothers have difficulty responding to these babies (perhaps because of their own depression that may be associated with a tendency to drink, and/or because of difficulties presented by the child), attachment security is compromised, and these alcohol-exposed children grow into preschoolers who show increased levels of depression at around 4 to 5 years of age. This has been shown in middleincome families, and even more clearly in low-income families who have additional risk factors that increase severity of this negative cycle. Additional analyses of middle-income families through age 6 revealed this process as most important for girls, but also found 
separate trends or significant direct effects of prenatal alcohol exposure on outcome in both boys and girls (O'Connor, 2001). Further research taking a developmental systems approach, and examining issues such as gender and socioeconomic differences, is crucial to creating targeted, empirically based early intervention for children who are alcoholexposed or have FASD diagnoses.

\section{Cumulative risk and FASD}

The impact of prenatal alcohol exposure is often coupled with that of other prenatal drug exposures, poor prenatal care, and life with parents who struggle with chemical dependency. Children may live in environments with health and safety concerns, relationship problems, and chaotic lifestyles. Longitudinal studies (eg, Arendt et al., 2004; Butz, Pulsifer, Leppert, Rimrodt, \& Belcher, 2003; Singer et al., 2004) have tracked the often negative developmental outcomes of children with prenatal substance exposures in these highrisk situations. Clinical studies have examined the difficult lives of children and the potential impact of intensive interventions through comprehensive chemical dependency treatment programs serving women with children (eg, Division of Alcohol \& Substance Abuse, 1999), long-term and very intensive family support programs for the highest risk mothers (eg, Grant, Ernst, Streissguth, \& Stark, 2005), and intensive early intervention with family support (eg, Butz et al., 2001; Nair, Schuler, Black, Kettinger, \& Harrington, 2003; Schuler, Nair, \& Kettinger, 2003). These studies are sharp reminders that children with FASD may often spend at least some of their early years in an environment of cumulative risk. A developmental systems approach emphasizes that cumulative risk could be especially harmful for a child made biologically vulnerable by prenatal alcohol exposure. With the implementation of CAPTA amendments and the IDEA Improvement Act of 2004, early intervention providers will more often be linked with the child welfare system and see children who not only experience cumulative risk but also have prenatal exposures. As a result, the need to refer children for FASD diagnosis or to refer those children who are diagnosed for appropriate supplementary services will increaseand specialized training and systematic linkages with service systems that work specifically with these families will be needed.

\section{TAKING ON THE CHALLENGE OF FASD IN EARLY INTERVENTION}

\section{A developmental systems model and its usefulness in guiding practice and research}

Developmental systems thinking can guide practice for young children with FASD or with prenatal alcohol exposure, and makes clear why early intervention for this population is especially important. A useful developmental systems model was created for early intervention with children who have special needs (Guralnick, 2001). Applying this model to FASD, prenatal alcohol exposure can be seen as placing a child at risk for (or creating) biological vulnerability and "disabling child characteristics," such as sensory sensitivities or unusual learning deficits. Such deficits are individually variable and arise in multiple domains of development (perhaps in subtle ways), and may emerge more clearly or become more debilitating over time, and these child deficits (and compensatory strengths) must be comprehensively assessed.

Disabling child characteristics can disrupt existing family interaction patterns, creating information and resource needs for parents, threats to parenting confidence, and both caregiver and family stress. Comprehensive assessment of these "environmental risk factors," and risks uniquely important in this population, are also essential. One unique risk factor identified through clinical experience centers around inappropriate caregiver reactions to deficits of the child with FASDdeficits caregivers may not easily recognize as a result of alcohol-induced CNS dysfunction, but see as willful disobedience. Other pivotal family risk factors have been documented in natural history research. For this population, 
past or current parental substance use, and poor quality of attachment security or poor quality of the childhood caregiving environment, is significantly associated with poor life outcomes (Streissguth et al., 2004). Protective factors hypothesized as important to this target population, such as the parent's level of optimism and parenting efficacy, use of specialized parenting practices, advocacy skill, and knowledge of FASD, or appropriate linkage to community resources (Olson et al., 2005), must also be assessed.

Early intervention should then aim to concurrently reduce the impact of disabling child characteristics and cumulative environmental risk, while at the same time enhancing protective factors. For the diagnosed child, a comprehensive intervention program can be developed and implemented, providing resource, social, and information supports, and direct intervention to enhance family interaction patterns, adjusted to the family's unique needs and targeted to the disability. For example, if a child with FASD has sensory sensitivities and processing speed deficits arising from prenatal alcohol exposure, then environmental modification and supportive OT services, caregiver education, and advocacy for later special education, might be necessary. Home visiting or clinical services to help caregivers understand alcohol-related brain damage, modify attitudes, learn specialized parenting skills, and learn effective advocacy to access existing community supports may be required when children with FASD have especially difficult behavior problems. Intervention models for such services have been developed and show promising findings in systematic research with somewhat older children with FASD (eg, Olson et al., 2005). For "at-risk" young children with prenatal alcohol exposure who may or may not yet show clinically concerning behaviors, preventative interventions are needed. For example, if attachment security is compromised in a very young child, intervention focused on enhancing secure attachment may be helpful-but may have to be adjusted for a biologically vulnerable child and the family structure (eg, foster, birth) in which the child is being raised. Early intervention models are being developed that incorporate a variety of child- and familyfocused intervention strategies as needed (eg, Coles, Kable, Dent, \& Lee, 2003; Gurwitch, Mulvihill, \& Chaffin, 2003).

Of course, research on FASD prevention and on biological methods to ameliorate the impact of prenatal alcohol exposure on child development (such as nutritional supplements) is important. But many children are born exposed to, and affected by, prenatal alcohol, so intervention research is essential. Developmental systems thinking can also guide intervention research. Specific "disabling child characteristics" of children with FASD (or prenatal alcohol exposure) must be identified. For deficits amenable to change, targeted intervention strategies must be studied. For example, young children with FASD or prenatal exposure likely have behavior regulation problems, and targeted interventions might involve teaching caregivers specialized techniques to calm their alcohol-exposed child or help the child self-sooth. It is also imperative to conduct research on the effectiveness of more comprehensive early intervention services. These reduce "environmental risk factors" that have already been identified and increase hypothesized protective parenting and family factors. Such research is already under way through, for example, collaborative research by several research groups funded by the Centers for Disease Control and Prevention (eg, Bertrand, Sidhu, \& Floyd, 2003).

\section{Educating early intervention professionals about FASD and improving service delivery}

To best serve children with FASD or prenatal substance exposure, what training is recommended for all early intervention professionals? As a start, this article presents important information about the disability. But there are additional needs for specialized preservice education and continuing education in the field, with training recommendations presented in Table 2. Training 
Table 2. Suggested training topics for early intervention providers working with children affected by parental substance abuse, including alcohol ${ }^{* \dagger} \dagger$

How to ask questions about prenatal exposure

The process of addiction and recovery; harm reduction and relapse prevention

How to provide FASD prevention information

How to recognize and screen for the characteristic facial features of FAS, small head size, and mild or greater growth impairment

Key behavioral symptoms signaling need for diagnostic referral when in the presence of alcohol exposure

Advocacy skills to help children at risk continue to be monitored or qualify for services even with subtle deficits, and to promote services in the next intervention setting

Neurodevelopmental disabilities, and specifics about FASD; how brain development and function are affected by prenatal alcohol exposure

How to provide appropriate early intervention given current data on FASD, with a focus on environmental modification and antecedent-based positive behavior support planning

The family experience of raising children with FASD, and differences between different family structures

How parental substance abuse affects children's lives

CAPTA and IDEA regulations, and interagency efforts to improve services for children affected by parental substance abuse

Systems of care for chemical dependency treatment, FASD diagnosis, child welfare, crisis placement, foster care, adoption, adult corrections, and adult developmental disabilities service.

*Contact Drs Carmichael Olson and Jirikowic for more information on training program.

${ }^{\dagger}$ FASD indicates fetal alcohol spectrum disorders; FAS, fetal alcohol syndrome; CAPTA, Child Abuse Prevention and Treatment Act; and IDEA, Individuals with Disabilities Education Act.

is an essential foundation to help providers develop skills to screen for FASD, guide families in obtaining a diagnosis, and effectively support the children with a diagnosis and their caregivers. Such training programs are under development and continuing education about some of these topics already exists. Beyond training, early intervention providers can build working relationships and formal connections with service provider systems that specialize in this area, such as woman-oriented chemical dependency treatment centers, FASD diagnostic clinics, FASD intervention programs, FASD parent support/advocacy organizations, and the child welfare system. At the state and local levels, interagency efforts can set up methods to raise awareness and train providers, facilitate smooth diagnostic and service referrals, modify categorical service requirements as needed, enhance collaborative care, and strengthen supports for these children and families.

\section{Screening for FASD}

Using a developmental systems approach, screening is an essential first step to identify children who should be referred for FASD diagnosis as young as possible. Screening should take place in all early intervention settings. The most efficient and effective screening method for FAS is to look for the characteristic facial features. Inexpensive and user-friendly tools are available (Lip-Philtrum Guides and FAS Facial Photographic Analysis Software) and have been used to effectively screen high-risk populations for FAS such as children in foster care (Astley, Stachowiak, Clarren, \& Clausen, 2002). Screening for ARND requires effective and appropriate inquiry about prenatal alcohol exposure because, unlike the FAS facial features, there 
is no pattern or type of CNS dysfunction that is specific to prenatal alcohol exposure and can clearly identify ARND. Providers should obtain training on how to ask about women's drinking and drug use, know their own agency guidelines before talking with caregivers about prenatal exposures, and remember that alcohol use at times other than pregnancy can suggest only the possibility of gestational alcohol exposure. Providers should also screen for behavior problems and growth impairment that occur in the presence of alcohol exposure.

\section{Helping families obtain an FASD diagnostic workup}

Early interventionists are well-placed to help families find a diagnostic center and obtain a workup for an identified child. Diagnostic services are most likely to be available in child guidance or child development centers, hospitals specializing in children's services, or the growing number of specialized FASD diagnostic clinics. The Substance Abuse and Mental Health Services Administration, a governmental agency, through its FASD Center for Excellence, has sponsored a Web site that as of 2007 collates all available information on diagnostic services, intervention, prevention efforts, screening programs, and research literature: http://fascenter.samhsa. gov/about/index.cfm.

\section{Understanding and supporting families through FASD diagnosis}

Early interventionists can play a key role in helping families adjust to the possibility that a child may have (or does have) FASD. Caregivers raising a child with FASD and behavior problems almost all experience high levels of child-related parenting stress (Olson et al., 2004). Perhaps more than with other neurodevelopmental disabilities, FASD carries with it an emotional overlay, because it is a birth defect that could have been preventedand the disruptive influence of addiction will, in some way, be part of the family history. Parents' emotional reactions to the diagnosis can range from anger, to refusal to believe or listen, to acceptance-but almost always include grief, and often a firm (but likely mistaken) conviction that all problems can be dealt with by nurturing parenting or extraordinary efforts at early intervention.

Reactions of birth parents are also influenced by whether parent(s) are currently drinking or involved in the recovery process. The emotions of guilt and shame, or the psychological defense of denial, can create barriers to problem recognition or offers of intervention. Continued drinking and/or accompanying illicit drug use can mean ineffective communication and unpredictable lives, and even raise ethical and legal dilemmas for providers. Working with birth parents requires interpersonal sensitivity, and at least some specialized training (or access to consultation with providers who understand the issues of addiction and recovery). But a basic precept is that meeting families where they are, with a respectful, open, nonjudgmental, and supportive stance, is the best response by providers to the feelings of families. To maintain such a stance, however, requires that an early intervention provider think through their emotional reactions to their own and others' substance use, and to the stark reality that parental alcohol use can compromise an affected child's lifelong development.

Foster families in many states often receive training on issues related to children's special needs, and even specifically about FASD. But a child's foster placement may be temporary, so early intervention providers often must support both foster parents and caregivers who may then receive the child, including birth parents in recovery. Adoption agencies now typically openly disclose to adoptive families what is known about a child's prenatal and postnatal risks and any special needs, and families may have specifically chosen to adopt a child with FASD. But because alcohol effects may reveal themselves over time, adoptive families often find themselves struggling as they realize what is really meant by a lifelong birth defect. Therefore, they 
may need intensive support from early interventionists. Furthermore, kinship adoptive placements may differ quite dramatically from nonrelative adoptive placements. Children in kinship placements are living in families touched directly by addiction. A maternal grandmother raising her grandson, for instance, might also be contending with her daughter's ongoing addiction, and the grief and shame (or anger) that arises from both the ongoing addictive behavior and its consequences that impact the child.

\section{Providing anticipatory guidance and a model for future intervention}

Early interventionists individualize and set up appropriate, clear structure and routines, promoting strengths and accommodating each child's particular deficits. Early intervention settings ideally aim to provide familycentered, integrated services that are based on a developmental framework and attempt to achieve inclusion (Guralnick, 2001). Such settings are, perhaps, a model for how children with FASD would best be served even after the early intervention period. It is a major challenge when a child with FASD transitions out of the early intervention system to the schools, and the child and family must then deal with the expectations of more independent function and reduced access to individualized programming that come with age. Early interventionists can smooth this transition. They can be the first to grasp the real hazards of prenatal alcohol exposure, referring children for diagnosis with FASD as early as possible, alerting caregivers to the growing child's likely emerging neurobehavioral deficits and increasing struggles with relationships, connecting families with needed additional services (such as alcohol treatment for parents)-and showing the family and the child's set of care providers what intervention strategies can really work. Early intervention providers can be proactive and essential gatekeepers and guides to the future.

\section{REFERENCES}

Adnams, C. M., Kodituwakku, P. W., Hay, A., Molteno, C. D., Viljoen, D., \& May, P. A. (2001). Patterns of cognitive-motor development in children with fetal alcohol syndrome from a community in South Africa. Alcoholism: Clinical and Experimental Research, 25(4), 557-562.

American Psychiatric Association. (1994). Diagnostic and statistical manual of mental disorders (4th ed.). Washington, DC: Author.

Arendt, R. E., Short, E. J., Singer, L. T., Minnes, S., Hewitt, J., Flynn, S., et al. (2004). Children prenatally exposed to cocaine: Developmental outcomes and environmental risks at seven years of age. Journal of Developmental \& Behavioral Pediatrics, 25(2), 83-90.

Astley, S. J. (2004). Diagnostic guide for fetal alcohol spectrum disorders: The 4-digit diagnostic code (3rd ed.). Seattle: University of Washington Publication Services.

Astley, S. J., \& Clarren, S. K. (2000). Diagnosing the full spectrum of fetal alcohol exposed individuals: Introducing the 4-Digit Diagnostic Code. Alcohol and Alcobolism, 4(35), 400-410.

Astley, S. J., Stachowiak, J., Clarren, S. K., \& Clausen, C. (2002). Application of the fetal alcohol syndrome facial photographic screening tool in a foster care population. Journal of Pediatrics, 141(5), 712-717.
Autti-Ramo, I., \& Granstrom, M. L. (1991). The psychomotor development during the first year of life of infants exposed to intrauterine alcohol of various duration. Neuropediatrics, 22, 59-64.

Barr, H. M., Streissguth, A. P., Darby, B. L., \& Sampson, P. D. (1990). Prenatal exposure to alcohol, caffeine, tobacco, and aspirin: Effects on fine and gross motor performance in 4-year-old children. Developmental Psychology, 26, 339-348.

Bertrand, J., Sidhu, J., \& Floyd R. L. (2003). Framework for diagnosis and intervention for children with fetal alcohol syndrome and their families. San Francisco: American Public Health Association. Retrieved April 3, 2006, from http://apha.confex.com/ apha/131am/techprogram/paper_59169.htm

Burden, M. J., Jacobson, S. W., \& Jacobson, J. L. (2005). Relation of prenatal alcohol exposure to cognitive processing speed and efficiency in childhood. Alcoholism: Clinical \& Experimental Research, 29(8), 1473-1483.

Butz, A. M., Pulsifer, M. B., Leppert, M., Rimrodt, S., \& Belcher, H. (2003). Comparison of intelligence, school readiness skills, and attention in in-utero drug-exposed and nonexposed preschool children. Clinical Pediatrics, 42(8), 727-739.

Butz, A. M., Pulsifer, M., Marano, N., Belcher, H., Lears, M. 
K., \& Royall, R. (2001). Effectiveness of a home intervention for perceived child behavioral problems and parenting stress in children with in-utero drug exposure. Archivers of Pediatric \& Adolescent Medicine, 155(9), 1029-1037.

Centers for Disease Control and Prevention. (2007). Retrieved February 14, 2007, from http://www.cdc.gov/ ncbdd/fas/fasak.htm

Chandler, L. S., Richardson, G. A., Gallagher, J. D., \& Day, N. L. (1996). Prenatal exposure to alcohol and marijuana: Effects on motor development of preschool children. Alcobolism: Clinical and Experimental Research, 20(3), 455-461.

Chudley, A. E., Conry, J., Cook, J. L., Loock, C., Rosales, T ., \& LeBlanc, N., (2005). Fetal alcohol spectrum disorder: Canadian guidelines for diagnosis. CMAJ, 172(5, Suppl). Retrieved June 13, 2005, from: http://www.cmaj.ca/cgi/content/full/172/5_suppl/ S1

Church, M. W., \& Kaltenbach, J. A. (1997). Hearing, speech, vestibular, and dentofacial disorders in fetal alcohol syndrome: A literature review. Alcoholism: Clinical and Experimental Research, 21, 495-512.

Coggins, T. E., Friet, T., \& Morgan, T. (1998). Analyzing narrative productions in older school-age children and adolescents with fetal alcohol syndrome: An experimental tool for clinical applications. Clinical and Linguistic Phonetics, 12(3), 221-236.

Coggins, T. E., Olswang, L. B., Carmichael Olson, H., \& Timler, G. R. (2003). On becoming socially competent communicators: The challenge for children with fetal alcohol exposure. In L. Abbeduto (Ed.), Language \& communication in mental retardation: International review in research on mental retardation (Vol. 27, pp. 121-150). New York: Academic Press

Coles, C. D., Kable, J., Dent, D., \& Lee. D. (2003). Sociocognitive habilitation with children with FAS. San Francisco: American Public Health Association. Retrieved April 3, 2006, from http://apha.confex.com/ apha/131am/techprogram/paper_59850.htm

Coles, C. D., Platzman, K. A., Raskind-Hood, C. L., Brown, R. T., Falek, A., \& Smith, I. E. (1997). A comparison of children affected by prenatal alcohol exposure and attention deficit hyperactivity disorder. Alcobolism: Clinical and Experimental Research, 21(1), 150-161.

Division of Alcohol \& Substance Abuse. (1999). Washington State MOMS Project: Perinatal Research and Demonstration Project (Final Report), National Institute on Drug Abuse. Olympia, WA: Department of Social and Health Services.

Dolk, H. (1991). The predictive value of microcephaly during the first year of life for mental retardation at seven years. Developmental Medicine \& Child Neurology, 33, 974-983.

Eyler, F. D., \& Behnke, M. (1999). Early development of infants exposed to drugs prenatally. Clinics in Perinatology, 26(1), 107-150.
Fried, P. A., \& Watkinson, B. (1990). 36- and 48-month neurobehavioral follow-up of children parentally exposed to marijuana, cigarettes and alcohol. Journal of Developmental and Behavioral Pediatrics, 11, 4958.

Fryer, S. L., McGee, C. L., Matt, G. E., Riley, E. P., \& Mattson, S. N. (2005). Evaluation of psychopathology in children with heavy prenatal alcohol to alcohol [Abstract 727]. Alcobolism: Clinical and Experimental Research, 29(5), 128A.

Goldschmidt, L., Richardson, G. A., Cornelius, M. D., \& Day, N. L. (2004). Prenatal marijuana and alcohol exposure and academic achievement at age 10. Neurotoxicology and Teratology, 26(4), 521-532.

Grant, T. M., Ernst, C. C., Streissguth, A., \& Stark, K. (2005). Preventing alcohol and drug exposed births in Washington State: Intervention findings from three parent-child assistance program sites. American Jour nal of Drug \& Alcohol Abuse, 31(3), 71-90.

Greene, T., Ernhart, C. B., Ager, J., Sokol, R., Martier, S., \& Boyd, T. (1991). Prenatal alcohol exposure and cognitive development in the preschool years. Neurotoxicology and Teratology, 13(1), 57-68.

Guralnick, M. (2001). A developmental systems model for early intervention. Infants and Young Children, 14(2), 1-18.

Gurwitch, R., Mulvihill, J., \& Chaffin, M. (2003). Prevention of secondary disabilities of children with Fetal Alcohol Syndrome/ARND. San Francisco: American Public Health Association. Retrieved April 3, 2006, from http://apha.confex.com/apha/ 131am/techprogram/paper_60858.htm

Howell, K. K., Lynch, M. E., Platzman, K. A., Smith, G. H., \& Coles, C. D. (2006). Prenatal alcohol exposure and ability, academic achievement and school functioning in adolescence: A longitudinal follow-up. Journal of Pediatric Psychology, 31(1), 116-126.

Individuals with Disabilities Improvement Act of 2004, 20 U.S.C.A $\S 1400$ et seq. (Thomson-West, 2005) [PL 10846].

Jacobson, J. L., Jacobson, S. W., Sokol, R. J., Martier, S. S., Ager, J. W., \& Kaplan-Estrin, M. G. (1993). Teratogenic effects of alcohol on infant development. Alcobolism: Clinical and Experimental Research, 17(1), 174-183.

Jacobson, S. W., Jacobson, J. L., \& Sokol, R. J. (1994). Effects of fetal alcohol exposure on infant reaction time. Alcoholism: Clinical and Experimental Research, 18(5), 1125-1132.

Jacobson, S. W., Stanton, A. M., Hay, M. J., Burden, M. J., Fuller, D. S., Roxford, J. A., et al. (2005). Impaired short delay eyeblink conditioning in children with FAS: Preliminary findings [Abstract 232]. Alcoholism: Clinical and Experimental Research, 29(Suppl. 5), 45A.

Janzen, L. A., Nanson, J. L., \& Block, G. W. (1995). Neuropsychological evaluation of preschoolers with fetal alcohol syndrome. Neurotoxicology and Teratology, 17(3), 273-279. 
Jirikowic, T. (2003). Sensory processing and integration in children with FAS and alcohol-related diagnoses: An exploratory study. Unpublished doctoral dissertation, University of Washington, Seattle.

Keeping Children and Families Safe Act of 2003, 42 U.S.C.A $\S 670$ et seq. (Thomson-West, 2003). [Child Abuse Prevention and Treatment Act (CAPTA) PL. 93247 as amended by Keeping Children and Families Safe Act of 2003 Pl 108-36].

Klintsova, A., Goodlet, C., \& Greenough, T. (1999). Therapeutic motor training ameliorates cerebellae effects of postnatal binge alcohol. Neurotoxicology and Teratology, 22, 125-132.

Larrouque, B., \& Kaminski, M. (1998). Prenatal alcohol exposure and development at preschool age: Main results from a French study. Alcoholism: Clinical and Experimental Research, 22(2), 295-303.

Larrouque, B., Kaminski, M., Dehaune, P., Subtil, D., Delfosse, M., \& Querleu, D. (1995). Moderate prenatal alcohol exposure and psychomotor development at preschool age. American Journal of Public Health, 85, 1654-1661.

Mattson, S. N., Lee, K. T., Hayden, K., \& Riley, E. P. (2005). Comparison of adaptive behavior deficits in children with fetal alcohol spectrum disorders and/or ADHD [Abstract 236]. Alcobolism: Clinical and Experimental Research, 29(5), 46A.

Mattson, S. J., \& Riley, E. P. (1998). A review of the neurobehavioral deficits in children with fetal alcohol syndrome or prenatal alcohol exposure. Alcoholism: Clinical and Experimental Research, 22(2), 279294.

Mattson, S. N., \& Riley, E. P. (2000). Parent ratings of behavior in children with heavy prenatal alcohol exposure and IQ-matched controls. Alcobolism: Clinical and Experimental Research, 24(2), 226231.

Mattson, S. N., Riley, E. P., Gramling, L., Delis, D. C., \& Jones, K. L. (1998). Neuropsychological comparison of alcohol exposed children with or without the physical features of fetal alcohol syndrome. Neuropsychology, 12(1), 16-153.

Miura, T., Whinery, L., Dominguez, H. D., Riley, E. P., \& Thomas, J. D. (2005). Exercise reduces the severity of behavioral alterations association with alcohol exposure during the brain growth spurt in rats [Abstract 735]. Alcoholism: Clinical and Experimental Research, 29(Suppl. 5), 130A.

Morse, B. A., Miller, P. T., \& Cermak, S. A. (1995). Sensory processing in children with FAS [Abstract]. $\mathrm{Al}$ coholism: Clinical and Experimental Research, 18, 503.

Murray, J., Olson, H., \& Montague, R. (2005). Psychiatric diagnosis and medication in fetal alcobol spectrum disorders. American Psychiatric Association. Retrieved from www.psych.org

Nair, P., Schuler, M. E., Black, M. M., Kettinger, L., \& Harrington, D. (2003). Cumulative environmental risk in substance abusing women: Early intervention, parenting stress, child abuse potential and child development. Child Abuse \& Neglect, 27(9), 997-1017.

National Center on Birth Defects and Developmental Disabilities. (2004). Fetal alcobol syndrome: Guidelines for referral and diagnosis. Washington, DC: Centers for Disease Control and Prevention. Retrieved March 1, 2006, from http://www.cdc.gov/ ncbddd/fas/documents/FAS_guidelines_accessible. pdf

National Institute on Alcohol Abuse and Alcoholism. (2000). Tenth special report to the U.S. Congress on alcobol and bealth: Highlights on current research. Washington, DC: U.S. Department of Health and Human Services, Public Health Service, National Institutes of Health. Retrieved July 2, 2005, from http://www.niaaa.nih.gov/publications/10 report/chap05.pdf

National Organization on Fetal Alcohol Syndrome. (2004). Historic agreement heralds new era for prevention and treatment of fetal alcohol spectrum disorders. Retrieved July 25, 2005, from www.nofas.org/ news/04152004.aspx

O'Connor, M. J. (2001). Prenatal alcohol exposure and infant negative affect as precursors of depressive features in children. Infant Mental Health Journal, 22(3), 291-299.

O' Connor, M. J., Kogan, N., \& Findlay, R. (2002a). Prenatal alcohol exposure and attachment behavior in children. Alcoholism: Clinical and Experimental Research, 26(10), 1592-1602.

O'Connor, M. J., Shah, B., Whaley, S., Cronin, P., Gunderson, B., \& Graham, J. (2002b). Psychiatric illness in a clinical sample of children with prenatal alcohol exposure. American Journal of Drug \& Alcobol Abuse, 28(4), 743-754.

Olson, H., Feldman, J. J., Streissguth, A. P., Sampson, P., \& Bookstein, F. L. (1998a). Neuropsychological deficits among adolescents with fetal alcohol syndrome: Clinical findings. Alcoholism: Clinical and Experimental Research, 22(9), 1998-2012.

Olson, H., Quamma, J., Brooks, A., Lehman, K., Ranna, M., \& Astley, S. (2005). Efficacy of a new model of behavioral consultation for families raising schoolaged children with FASD and behavior problems [Abstract 243]. Alcoholism: Clinical and Experimental Research, 29(Suppl. 5), 47A.

Olson, H. C. (1994). The effects of prenatal alcohol exposure on child development. Infants and Young Children, 6(3), 10-25.

Olson, H. C., Brooks, A., Davis, C., \& Astley, S. (2004). Creating and testing a new model of behavioral consultation for families raising school-aged children with FAS/ARND and behavioral problems. Alcoholism: Clinical and Experimental Research, 28(suppl 5), 718.

Olson, H. C., Morse, B., \& Huffine, C. (1998b). Development and psychopathology: Fetal alcohol syndrome 
and related conditions. Seminars in Clinical Neuropsychiatry, 3(4), 262-284.

Olson, H. C., Streissguth, A. P., Sampson, P. O., Barr, H. M., Bookstein, F. L., \& Thiede, K. (1997). Association of prenatal alcohol exposure with behavioral and learning problems in early adolescence. Journal of the American Academy of Child and Adolescent Psychiatry, 36(9), 1187-1194.

Osborn, J. A., Harris, S. R., \& Weinberg, J. (1993). Fetal alcohol syndrome: Review of the literature with implications for physical therapists. Physical Therapy, 73(9), 599-607.

Richardson, G. A., Day, N. L., \& Goldschmidt, L. (1995). Prenatal alcohol, marijuana, and tobacco use: Infant mental and motor development. Neurotoxicology and Teratology, 17(4), 479-487.

Roebuck, M. S., \& Riley, E. P. (1999). Behavioral and psychosocial profiles of alcohol-exposed children. Alcoholism: Clinical and Experimental Research, 23(6), 1070-1076.

Schuler, M. E., Nair, P., \& Kettinger, L. (2003). Drugexposed infants and developmental outcome: Effects of a home intervention and ongoing maternal drug use. Archives of Pediatric \& Adolescent Medicine, 157(2), 133-138.

Singer, L. T., Minnes, S., Short, E., Arendt, R., Farkas, K., Lewis, B., et al. (2004). Cognitive outcomes of preschool children with prenatal cocaine exposure. Journal of the American Medical Association, 291(20), 2448-2456.

Steinhausen, H. C., \& Spohr, H. L. (1998). Long-term outcome of children with fetal alcohol syndrome: Psychopathology, behavior and intelligence. Alcoholism: Clinical and Experimental Research, 22(2), 334338.

Stratton, K., Howe, C., \& Battaglia, F. (Eds.). (1996). Fetal alcohol syndrome: Diagnosis, epidemiology, prevention and treatment. Washington, DC: National Academy Press.

Streissguth, A. (1997). Fetal alcobol syndrome: A guide for families and communities. Baltimore, MD: Paul H. Brookes.

Streissguth, A., Barr, H., Kogan, J., \& Bookstein, F. L. (1996). Understanding the occurrence of secondary disabilities in clients with fetal alcobol syndrome (FAS) and fetal alcohol effects (FAE). Seattle: University of Washington Publications Services.

Streissguth, A. P., Barr, H. M., Martin, D. C., \& Herman, C. S. (1980). Effects of maternal alcohol, nicotine, and caffeine use during pregnancy on infant mental and motor development at eight months. Alcoholism: Clinical and Experimental Research, 4(2), 152-164. Streissguth, A., Barr, H. M., Sampson, P. D., \& Bookstein, F. L. (1994). Prenatal alcohol and offspring development: The first fourteen years. Drug and Alcohol Dependence, 36, 89-99.

Streissguth, A. P., Barr, H. M., Sampson, P. D., Darby, B. L., \& Martin, D. C. (1989). IQ at age four in relation to maternal alcohol use and smoking during pregnancy. Developmental Psychology, 25, 3-11.

Streissguth, A. P., Bookstein, F. L., Barr, H. M., Sampson, P. D., O'Malley, K., \& Young, J. K. (2004). Risk factors for adverse life outcomes for fetal alcohol syndrome and fetal alcohol effects. Journal of Developmental and Behavioral Pediatrics, 25(4), 228-238.

Streissguth, A. P., Bookstein, F. L., Sampson, P. D., \& Barr, H. M. (1993). The enduring effects of prenatal alcobol exposure on child development: Birth through seven years, a partial least squares solution. Ann Arbor: University of Michigan Press.

Streissguth, A. P., Bookstein, F. L., Sampson, P. D., \& Barr, H. M. (1995). Attention: Prenatal alcohol and continuities of vigilance and attentional problems from 4 through 14 years. Development and Psychopathology, 7(3), 419-446.

Surgeon General's Advisory on Drinking and Pregnancy. (2005, February). U.S. Surgeon General releases advisory on alcohol use in pregnancy. Retrieved July 1, 2005, from http://www.surgeongeneral.gov/ pressreleases/sg02222005.html

Thomas, S. E., Kelly, S. J., Mattson, S. N., \& Riley, E. P. (1998). Comparison of social abilities of children with fetal alcohol syndrome to those of children with similar IQ scores and normal controls. Alcobolism: Clinical and Experimental Research, 22(2), 528-533.

Whaley, S. E., O'Connor, M. J., \& Gunderson, B. (2001). Comparison of the adaptive functioning of children prenatally exposed to alcohol to a non-exposed clinical sample. Alcoholism: Clinical and Experimental Research, 25(7), 1018-1024.

World Health Organization. (2005). ICD-10: International statistical classification of diseases and related bealth problems (10th Rev., 2nd ed.). Geneva: Author.

Zero to Three. (2005). DC:O-3R: Diagnostic classification of mental bealth and developmental disorders of infancy and early childbood DC:O-3R revision task force. Washington, DC: Zero to Three Press. 